
characterization of non-tumorigenic and

SUBJECT AREAS:

BIOPHYSICS

CANCER

ENGINEERING

BIOTECHNOLOGY

Received

1 August 2012

Accepted

7 January 2013

Published

26 April 2013

Correspondence and requests for materials should be addressed to D.W. (wirtz@jhu.edu)

*A comprehensive list of authors and affiliations appear at the end of the paper. \section{metastatic cells}

\author{
The Physical Sciences - Oncology Centers Network*
}

To investigate the transition from non-cancerous to metastatic from a physical sciences perspective, the Physical Sciences-Oncology Centers (PS-OC) Network performed molecular and biophysical comparative studies of the non-tumorigenic MCF-10A and metastatic MDA-MB-231 breast epithelial cell lines, commonly used as models of cancer metastasis. Experiments were performed in 20 laboratories from 12 PS-OCs. Each laboratory was supplied with identical aliquots and common reagents and culture protocols. Analyses of these measurements revealed dramatic differences in their mechanics, migration, adhesion, oxygen response, and proteomic profiles. Model-based multi-omics approaches identified key differences between these cells' regulatory networks involved in morphology and survival. These results provide a multifaceted description of cellular parameters of two widely used cell lines and demonstrate the value of the PS-OC Network approach for integration of diverse experimental observations to elucidate the phenotypes associated with cancer metastasis.

T he conversion from a non-tumorigenic state to a metastatic one is of critical interest in cancer cell biology, as most deaths from cancer occur due to metastasis ${ }^{1}$. Typically, we think of the activation of metastasis as one of the hallmarks of cancer ${ }^{2}$ and as a highly regulated, multistep process defined by a loss of cell adhesion due to reduced expression of cell adhesion molecules such as E-cadherin, degradation of the extracellular matrix (ECM), conversion to a motile phenotype, vascular infiltration, exit and colonization to a new organ site (i.e., intra- and extravasation), dormancy, and re-activation. From a physical sciences perspective, metastasis can be viewed as a "phase" transition, albeit occuring far from thermodynamic equilibrium ${ }^{3}$. Though this transition has been the focus of much cancer biology research, there is still an incomplete understanding of this phase change, in particular, the physical biology of the metastatic state of a cell compared to its pre-malignant state. Understanding the physical forces that metastatic cells experience and overcome in their microenvironment may improve our ability to target this key step in tumor progression.

The newly formed Physical Sciences-Oncology Centers (PS-OC) Network, sponsored by and under the auspices of the Office of Physical Sciences-Oncology at the National Cancer Institute (OPSO/NCI), is a multidisciplinary network of twelve research centers across the US formed, in part, to test the fundamental hypothesis that physical processes (e.g., mechanics, dynamics) play a critical role in cancer initiation and metastasis. The PSOC Network brings analytic techniques and perspectives from the physical sciences to the interpretation of biological data and consists of physicists, engineers, mathematicians, chemists, cancer biologists, and computational scientists. The goal of the PS-OC Network is to better understand the physical and chemical forces that shape and govern the emergence and behavior of cancer at all length scales. The study described in this manuscript focused on physical changes associated with metastasis. A controlled set of comparative studies of two cell lines that are extensively used as cell models of cancer metastasis and straddle the metastatic transition was undertaken by the PS-OC Network.

The cell lines analyzed were the immortalized human breast epithelial cell line MCF-10A, representing a nontumorigenic state, and the human metastatic breast cell line MDA-MB-231, representing a malignant state. Distinguishing features of the adherent, non-transformed, MCF-10A cells are their lack of tumorigenicity in nude mice, lack of anchorage-independent growth, and dependence on growth factors ${ }^{4}$. In contrast, MDA-MB231 cells $^{5}$ form highly malignant, invasive tumors in vivo, are resistant to chemotherapy drugs such as paclitaxel, exhibit anchorage-independent growth, and grow independently of growth factors. Although MCF-10A cells have wild-type 53 and MDA-MB-231 cells have mutant p53, both cell lines are negative for the estrogen receptor (ER), progesterone receptor (PR), and human epidermal growth factor receptor 2 (HER2) ${ }^{6,7}$.

To ensure that data generated across the multiple PS-OC laboratories could be integrated, culture guidelines, common culture reagents, and the two fully characterized, karyotyped cell lines were distributed to PS-OC laboratories. This minimized phenotypic and genotypic drift. After demonstration of growth uniformity, the 
cells were evaluated by a battery of physical measurements, as outlined in Table 1, encompassing complementary physical, biochemical, and molecular assays, to establish a metastatic signature across multiple length scales, including the molecular, subcellular, cellular, and tumor length scales. Novel biophysical techniques interrogated classic phenotypic 'hallmark' properties of the two cell lines (e.g., morphology, motility, stress responses) and physical cell properties (e.g., shear rheology). A novel model-based regulatory network approach was used to generate hypotheses of linkages between molecular and physical signatures of the cell lines. By interrogating this one-of-a-kind dataset, this pilot study provides insight into intrinsic differences in the physical properties of metastatic cancer cells $v s$. their non-tumorigenic counterparts, while demonstrating the importance of the technologies employed from the physical sciences and the value of a network approach to the study of cancer biology.

\section{Results}

In order to generate integrated data across the PS-OC Network, the MCF-10A and MDA-MB-231 cell lines were characterized, expanded, and distributed to each PS-OC with common protocols and reagents to standardize culturing procedures for each cell line (Suppl. Fig. 1a). Each PS-OC expanded the cells and was required to submit predetermined annotations that included phase-contrast microscopic images at specified passage numbers, seeding densities, and culturing times to ensure phenotypic uniformity of starting material (Suppl. Fig. 1b). Each of the laboratories then initiated experiments using different methodologies to explore cell morphology, motility and mechanics, stress responses and survival (drug and hypoxia), and molecular networks.

Comparative cancer cell morphology. Cancerous cells are often described as having an altered appearance and morphology; cancer pathologists and oncologists routinely use cell and nuclear morphology to stage cancer and propose treatments. Clinical studies have linked properties of tumor cell mass and patient survival to variations in individual cells ${ }^{8}$ and nuclear morphology 9 . In particular, breast cancer cell line morphologies have been correlated to invasiveness and gene expression profile ${ }^{10}$. In this study, morphological parameters of the MCF-10A and MDA-MB-231 cells, including cell and nuclear shape, nuclear disorder strength, membrane lipid raft abundance, and cell growth in response to matrix stiffness were characterized. To determine the two- (2D) and three-dimensional (3D) properties of the two cell lines, MCF-10A and MDA-MB-231 cells were grown as monolayers and imaged in 2D by differential interference contrast microscopy (Fig. 1a). The MDA-MB-231 cells had a higher width-to-length aspect ratio of 1:1.77 compared with the smaller, rounder MCF-10A cells' width-to-length aspect ratio of 1:1.39 (Fig. 1a). Three-dimensional cellular and nuclear shapes were determined using single-cell optical computed tomography and volumetric image analysis ${ }^{11}$. Representative pseudo-color renderings of $3 \mathrm{D}$ cell volumes are shown in Fig. 1b. Detached MDA-MB-231 cells exhibited consistent kidney-bean shaped nuclei with a relatively consistent extent of concavity, whereas nuclei of MCF-10A cells were more heterogeneous in shape. Nuclear sphericity parameters calculated from phase-contrast images were 1.42 and 1.39 for the MDA-MB-231 and MCF-10A cells, respectively, demonstrating the significantly non-spherical shapes of the nuclei of both cell types (Fig. 1b iii). This is contrary to the popular notion of suspended cells having spherical nuclei and is a novel observation of nuclear shape for these two otherwise wellcharacterized cell lines.

Partial wave spectroscopic (PWS) microscopy is a recently introduced high-resolution approach to characterize cancer cell morphology and the nanoscale architecture of the nucleus ${ }^{12}$. PWS employs back-scattered light to quantify the spatial variations of the refractive index or macromolecular mass density. Measurements of macromolecular compaction are reported as the nuclear disorder strength, $L_{\mathrm{d}}$, where an increase in the disorder strength of the cell nanoarchitecture is used as an indicator of early events in carcinogenesis ${ }^{12}$. Nuclear regions of the MDA-MB-231 and MCF-10A cell lines were analyzed and $L_{\mathrm{d}}$ calculated for each cell line and normalized to MCF$10 \mathrm{~A}$ values as baseline, as illustrated in Fig. 1c (PWS images are shown on the right and the bright field reflectance (BFR) images, from which PWS images were computed, are on the left). Metastatic MDA-MB-231 cells exhibited a statistically significant $(\mathrm{p}<0.0004)$ $80 \%$ increase in nuclear disorder strength relative to the non-tumorigenic MCF-10A cells. This difference in nuclear $L_{\mathrm{d}}$ indicates that the

Table 1 | Cell physical parameters, methods and measurements

Cell physical parameter Technology name/ Physical method

Measurement

Morphology

Cell and nuclear shape, volume

Nuclear architecture

Cell growth as a function of matrix stiffness

Cell surface
2D: Differential interference contrast microscopy

3D: Optical computed tomography

Partial wave spectroscopy

Immunofluorescence; confocal microscopy

Total internal reflection fluorescence; epifluorescence microscopy
2D: Shape; length

3D: Volume; characteristic nuclear shape (nuclear sphericity parameter)

Nuclear disorder strength $\left(L_{d}\right)$

Cell proliferation; cell morphology

CD44 expression patterns; lipid raft distribution

\section{Motility and Mechanics}

\section{Cell motility}

Cell adhesion and rolling

Mechanical flexibility

Internal fluidity

Endogenous force generation
1D, 2D, and 3D motility assays

HA Micropatterns; flow chamber adhesion assay

Atomic force microscope-based nano-indentation

Ballistic injection nanorheology

Traction force microscopy

\author{
Speed; radial displacement \\ Cell binding to HA pattern; rolling velocity \\ Elastic modulus \\ Mean square displacement \\ Tension maps, force magnitudes
}

Stress Response and Survival

\begin{tabular}{lll}
\hline $\begin{array}{l}\text { Hypoxic conditions } \\
\text { Chemical stress }\end{array}$ & $\begin{array}{c}\text { Microscopic imaging of 3D cultures (intracellular } \mathrm{Ca}^{2+} \\
\text { and DNA) }\end{array}$ & $\begin{array}{c}\text { 2D Cell viability; 3D O } \mathrm{O}_{2} \text { consumption; CEA } \\
\text { expression } \\
\text { Cell viability }\end{array}$
\end{tabular}

Abbreviations: 2D: 2-dimensional; 3D: 3-dimensional; CEA, carcinoembryonic antigen; HA, hyaluronic acid.

Footnote: Additional PS-OC methods are noted in the results and given in the supplementary information. 
MCF-10A

a

DIC

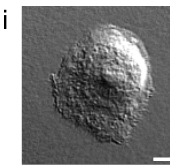

b

3D

cytometry

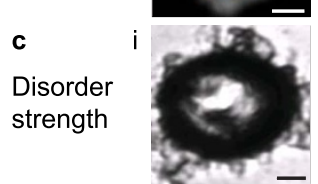

BFR

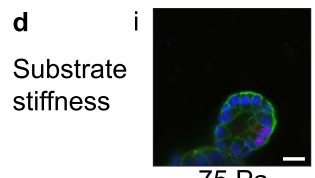

$75 \mathrm{~Pa}$

e

CD44 distribution

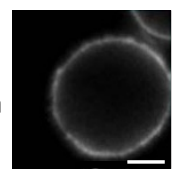

EFM

f

Lipid raft distribution

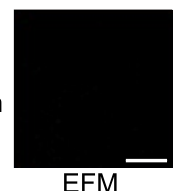

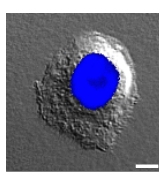
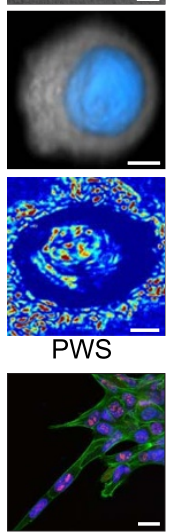

$6,000 \mathrm{~Pa}$

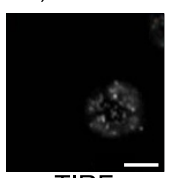

TIRF

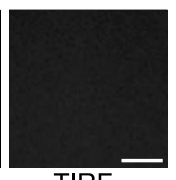

TIRF

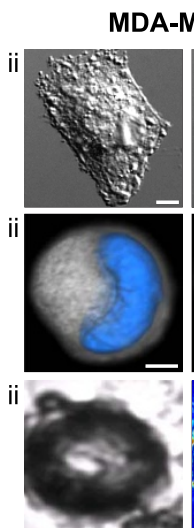

BFR

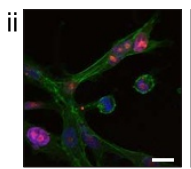

$75 \mathrm{~Pa}$

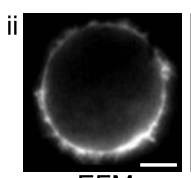

EFM

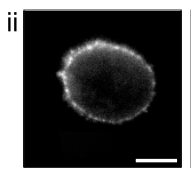

EFM
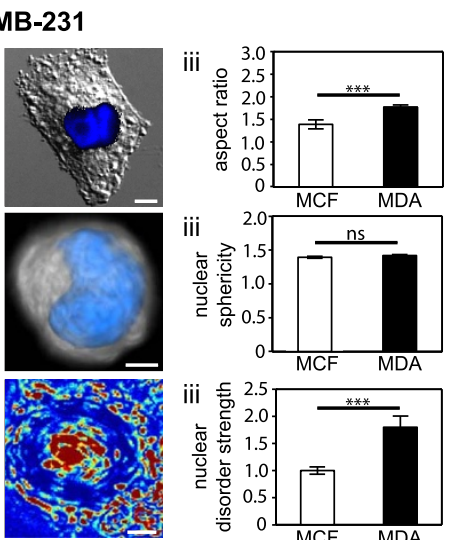

PWS
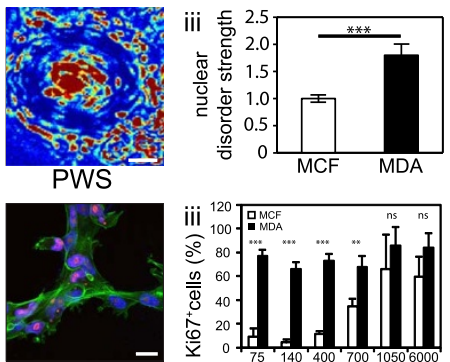

6,000 Pa

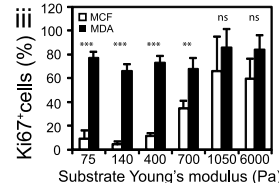

Substrate Young's modulus ( $\mathrm{Pa}$ )

iii

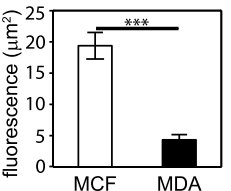

TIRF

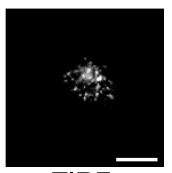

TIRF

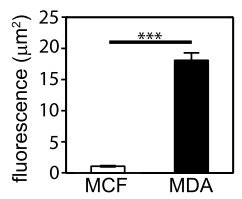

Figure 1 Comparative cell morphology. (a) Differential interference contrast (DIC) microscopy. (i, ii) Left: Volume rendering from DIC micrographs of each cell type (gray, H\&E stained). Right: Same as left with EFM images of DAPI stained nuclei (blue) superposed. (iii) Aspect ratios of cell bodies (mean \pm s.e.m.). (b) 3D cytometry. (i,ii) Pseudo-colored volume rendering of suspended and fixed H\&E stained cells imaged by optical cell CT. Cytoplasm is grey and nucleus is blue. (iii) Nuclear sphericity of the cell nuclei (mean \pm s.e.m.). (c) Nuclear disorder strength. (i, ii) Left: Bright field reflectance (BFR) images. Right: PWS microscopic images. Color shows the magnitude of the nuclear disorder strength $\left(\mathrm{L}_{\mathrm{d}}\right)$ (low: blue, high: red). $\mathrm{L}_{\mathrm{d}}$ values normalized to 1.0 for MCF-10A cells. (iii) Nuclear disorder strength (mean \pm s.e.m.). (d) Substrate stiffness. (i, ii) Confocal immunofluorescence of cells grown for 15 days in 3D on soft (Left, $75 \mathrm{~Pa}$ ) and hard (Right, $6000 \mathrm{~Pa}$ ) reconstituted basement membrane-conjugated polyacrylamide gel matrix. Cells stained for Ki-67 cell proliferation marker (red) and DNA using DAPI. (iii) Fraction of Ki-67 positive cells as function of substrate stiffness (mean \pm s.e.m.). (e) CD44 distribution. (i, ii) CD44 distribution visualized by anti-CD44 antibodies using (Left) epifluorescence microscopy (EFM) and (Right) total internal reflection fluorescence (TIRF). (iii) Fluorescent area in $\mu \mathrm{m}^{2}$ calculated from TIRF images (mean \pm s.e.m.). Bar graph compares significant difference in immunofluorescence intensity between TIRF images for MDA and MCF cells, not the EFM images. (f) Lipid raft distribution. (i, ii) Left: Lipid raft distribution visualized by anti-CT-B antibodies using EFM. Right: raft distribution visualized by anti-CT-B antibodies using TIRF. (iii) Fluorescent area in $\mu \mathrm{m}^{2}$ calculated from TIRF images (mean \pm s.e.m.). All scale bars are $5 \mu \mathrm{m}$. All $p$-values are indicated according to the Michelin guide scale ( $p \leq 0.001$ : [***]; $0.001<p \leq 0.01$ : [**]; $0.01<p \leq 0.05$ : $\left[{ }^{*}\right] ; 0.05<p$ : ns).

metastatic and non-tumorigenic cells have very different nuclear architectures.

There is increasing evidence of a functional relationship between tissue rigidity and tumor progression; indeed, tumors are often stiffer than normal tissues ${ }^{13}$. An investigation of whether cell matrix stiffness modulates cell growth and morphology was carried out with the two cell lines using ECM-crosslinked polyacrylamide gels of varying stiffness (Fig. 1d). The substrate stiffness was measured by shear rheology (the study of the flow of matter) ${ }^{14}$. When MCF-10A cells were grown for more than 15 days on a soft matrix (with substrate stiffness of either $75 \mathrm{~Pa}$ or $140 \mathrm{~Pa}$ ), the cells formed welldifferentiated, hollow, growth-arrested acini. When the cell matrix stiffness was increased to $6000 \mathrm{~Pa}$, the stiffer substrate induced an increase in cell growth but cells formed poorly differentiated monolayers (Fig. 1d) with nearly $70 \%$ of cells highly proliferative as evidenced by Ki-67 positive-staining. Conversely, MDA-MB-231 cells exhibited similar morphology and growth rate regardless of substrate, suggesting that these cells have a significantly reduced mechano-sensitivity. This insensitivity to matrix stiffness may be beneficial during metastasis as metastatic cells encounter different substrates during the dissemination process, and the lack of sensitivity to matrix stiffness allows these cells to proliferate in a wide variety of environments.

The expression and distribution of cell surface glycoproteins are a major determinant of cellular response to the microenvironment ${ }^{15}$. One of these glycoproteins, CD44, is a hyaluronic acid (HA) receptor involved in cell-cell adhesion and cell-matrix interactions, which are critical to the metastatic process. Variant isoforms have been associated with cancer metastasis, particularly in tumors originating from epithelia, including breast cancer ${ }^{16,17}$. Investigation of the surface receptor expression on MCF-10A and MDA-MB-231 cells using epifluorescence microscopy (EFM) images (Fig. 1e) revealed that the macroscopic CD44 distributions of the two cell lines did not differ significantly $(\mathrm{p}>0.75)$ (data not shown). This confirms a previous report using confocal microscopy, which showed a similar localization of CD44 on MDA-MB-231 cells $^{18}$. In contrast, total internal reflection fluorescence (TIRF) images of the same cells (Fig. 1e) indicated that CD44 was abundantly present at the point 
of contact between the cell membrane and the substrate surface in the MCF-10A but was observed at markedly lower levels (by a factor of 4.5) in the MDA-MB-231 cells (Fig. 1e iii). Interestingly, CD44 expression and distribution were not affected in either MCF-10A or MDA-MB-231 cells when cells were grown in 3D on substrates of differing stiffness. CD44 was mainly present at cell-cell junctions and was evenly distributed along the plasma membrane in both cell types (data not shown). When the cells were treated with a fluorescent probe specific to membrane lipid rafts, enhanced labeling of the MDA-MB-231 cells relative to MCF-10A cells was observed (Fig. 1f), suggesting the presence of lipid rafts only in the metastatic breast cancer cells. This finding is consistent with reports of elevated levels of cholesterol-rich lipid rafts in breast and prostate cancer cells compared to their normal counterparts ${ }^{17}$. Taken together, these experiments suggest that although the general topography of the two cell lines are similar, the surface presentation of the cell adhesion protein CD44 and formation of lipid raft domains containing additional cell surface receptors are significantly different.

Comparative cancer cell motility and mechanics. Cell migration. One of the distinguishing hallmarks of metastatic cells is their capacity to steer through multiple physical microenvironments such as the ECM of the stromal space and, following intravasation, along vasculature walls. Traditionally cell motility studies have been performed in 2D environments (i.e., flat substrates). Here, cell motility was evaluated in one-dimensional (1D), 2D, and 3D environments ${ }^{19-21}$. These multidimensional experiments revealed that regulation of cell speed and maximum displacement were critically dependent on the dimensionality of the environment. In a $1 \mathrm{D}$ environment where a cell is constrained to move only forward or backward ( $13 \mu \mathrm{m}$ wide by $25 \mu \mathrm{m}$ deep silicon-etched fibronectincoated channels), MCF-10A cells traveled more than three times faster than MDA-MB-231 cells (Fig. 2a iii). However, consistent with their metastatic potential, MDA-MB-231 cells traveled farther (based on radial displacement from their original position) than MCF-10A cells along the same $1 \mathrm{D}$ channels (Fig. 2a iii). In a 2D environment, MCF-10A cells moved in a circular or pin-wheel style of motility - the leading edge swung in an arc while the lagging edge often remained pinned in place (data not shown). MDA-MB-231 cells moved more linearly, though more slowly, and MCF-10A cells were found to travel farther than MDA-MB-231 cells on the 2D collagen matrix (Fig. 2a iii). MCF-10A cells also exhibited slightly faster migration in a wound healing assay (see Suppl. Fig. 5). MCF-10A cells also moved in a circular motion around the $3 \mathrm{D}$ void in which they were embedded, but MDA-MB-231 cells again traveled farther than MCF-10A cells when embedded inside 3D collagen matrices (data not shown). Thus, given the dimensional constraints of the cellular environment, the non-tumorigenic cells tended to move faster than the metastatic cells, but remained within a limited circular area, whereas MDA-MB-231 cell motility was linear and did not exhibit the same distance limitations.

ECM matrix components are known to potentiate breast tumor metastasis by enhancing cell invasion ${ }^{22}$, therefore, the functional relationship between cell motility and ECM molecules such as laminin (a primary ECM component of the basement membrane in breast tissue), hyaluronic acid (HA, an anionic non-sulfated glycosaminoglycan spatially distributed in the ECM), and cell surface adhesion molecule E-selectin were investigated. Traction force microscopy (TFM) was used to measure the pulling forces exerted by single cells on substrates with different concentrations of laminin. MDA-MB-231 cells generated more traction forces than MCF-10A cells at all tested laminin concentrations $(0.1 \mu \mathrm{g} / \mathrm{ml}, 10 \mu \mathrm{g} / \mathrm{ml}$, and $50 \mu \mathrm{g} / \mathrm{ml}$ ) (Fig. 2b iii, intermediate concentration data not shown), suggesting that laminin interactions may play a role in promoting breast tumor cell aggressiveness. Cell adhesion to HA substrates and CD44 expression in the two cell types were compared by growing cells on micropatterned arrays with covalently linked HA (Fig. 2c). Immunofluorescence and flow cytometry experiments confirmed that MDA-MB-231 cells had approximately two-fold more CD44 expression than MCF-10A cells (Fig. 2e iii). Nevertheless, MCF-10A cells adhered preferentially to HA, whereas MDA-MB231 cells showed no preference (Fig. 2c iii). The lack of HA adhesion preference of the metastatic cells is likely due to the lack of CD44 in the membrane. This is evident in the TIRF data (Fig. 1e iii). Whereas the EFM analysis (data not shown) shows 1:1 bulk CD44 levels, by TIRF, levels of CD44 are 2:1, a difference presumably due to the presence/absence of external HA in the environment.

The comparative role of E-selectin in the cell migration of metastatic and non-malignant cells may play a critical role in the ability to adhere to vasculature walls for efficient extravasation to secondary organs ${ }^{23}$. For example, in trans-endothelial migration of circulating tumor cells (CTC), endothelial cell surface ligands increase the adhesive forces and hence the residence time and extravasation of CTCs from the circulatory system. Fluid shear forces due to blood flow can counteract these forces. Shear stresses from 1-4 dyn $/ \mathrm{cm}^{2}$ are typical of veins, whereas higher stresses ranging from $4-30 \mathrm{dyn} / \mathrm{cm}^{2}$ can occur in arteries ${ }^{1}$. Here, flow-chamber experiments showed that MDA-MB-231 cells neither adhered nor rolled on either $5 \mu \mathrm{g} / \mathrm{mm}^{2}$ or $10 \mu \mathrm{g} / \mathrm{mm}^{2}$ E-selectin-coated surfaces over a range of physiological wall shear stresses from $1-8 \mathrm{dyn} / \mathrm{cm}^{2}$ (data not shown). In contrast, MCF-10A cells adhered and rolled on $5 \mu \mathrm{g} / \mathrm{mm}^{2}$ E-selectincoated surfaces over the entire range of stresses ranging from 1 to $8 \mathrm{dyn} / \mathrm{cm}^{2}$ (Fig. 2d iii). These results differ from those of Zen et al. ${ }^{18}$, who found that MDA-MB-231 cells were able to traverse a model endothelial monolayer in the absence of shear forces in a CD44- and E-selectin-dependent manner ${ }^{17}$. Given the expression of CD44 on MDA-MB-231 cells, the CD44/E-selectin binding interaction (affinity and/or expression) on the cell surface may be insufficient to increase the residence time in the presence of significant shear forces, and other ECM ligands or adhesion molecules may compensate in vivo. In spite of an apparent lack of $\mathrm{HA}$ and E-selectin binding by MDA-MB-231 cells, both MDA-MB-231 and MCF-10A cells exhibited an ECM deposition similar to that of Nuff fibroblast cells (data not shown), however the ECM structures appear to be distinct (Fig. 2e i).

Cell mechanics. Consistent with observations of amoeboid movement of invasive cancer cells, the ability of a cell to move through multiple tissue compartments, often via small portals, relies on amoeba-like deformability. The mechanical deformability of MCF$10 \mathrm{~A}$ and MDA-MB-231 cells was measured by an atomic force microscope (AFM) aligned with a confocal fluorescence microscope lens (AFM-CLSM) for fluorescence lifetime imaging measurements (FLIM) (Fig. 2f) ${ }^{24}$. Cells stained with nuclear and nucleolar dyes were indented at distinct points over the lamella, nucleus, and nucleoli. The resulting force-indentation curves were fitted to a modified Hertz model in 100-nm intervals, yielding depth-dependent elastic moduli. At shallow indentation depths, the two cell lines had similar elastic moduli $(\sim 200 \mathrm{~Pa})$. Cytoplasmic and nuclear stiffness of MDA-MB-231 cells increased only slightly with increasing indentation depth, whereas cytoplasmic and nuclear stiffness of MCF-10A cells both increased - though with different strain-hardening profiles - about four-fold to an elastic modulus of $\sim 1.6 \mathrm{kPa}$. At indentation points over nucleoli, both cell lines showed stiffening with increasing depth, with MDA-MB-231 and MCF-10A cells rising to elastic moduli of $\sim 1 \mathrm{kPa}$ and $\sim 1.5 \mathrm{kPa}$, respectively, at $0.8 \mu \mathrm{m}$. These findings suggest that mechanical loads are transduced through the cytoskeleton differently in the two cell lines. The decreased elastic modulus and increased deformability of MDA-MB-231 cells is consistent with their ability to traverse narrow matrices.

Cytoplasmic viscoelasticity is also an important feature of amoeboid movement. Subcellular viscoelasticity was investigated by ballistic injection nanorheology (BIN; movement of nanoparticles in a 

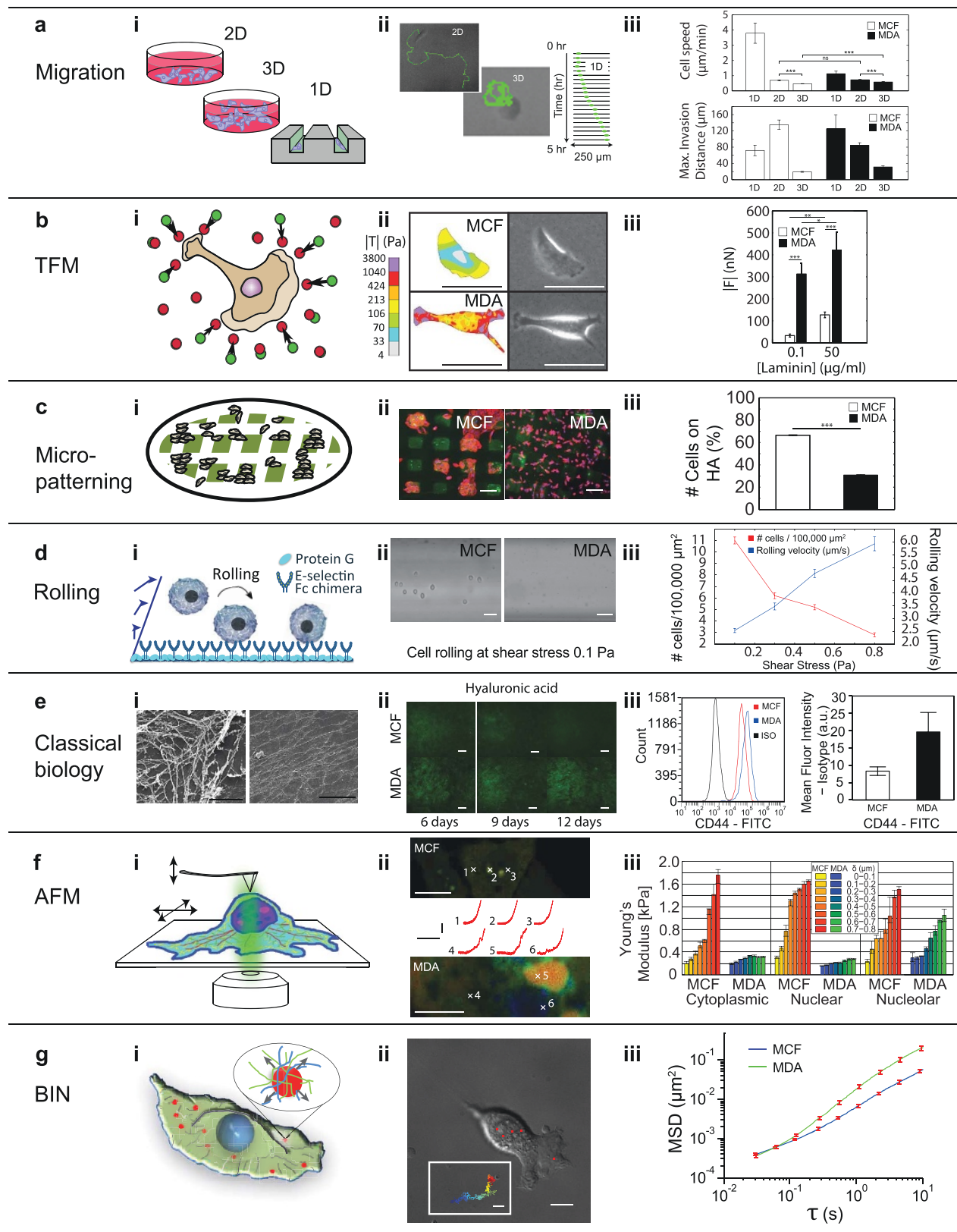

Figure 2 Motility and mechanics. (a) Three substrates used to monitor cell motility: 2D collagen-coated glass substrate, 3D collagen matrix, and 1D fibronectin-coated microchannels (13 $\mu \mathrm{m}$ wide, $25 \mu \mathrm{m}$ deep) etched in silicon. Motion tracking based on time-lapse imaging. (i) Schematic. (ii) Tracking data. (iii) Speed $(\mu \mathrm{m} / \mathrm{min}$ ) and maximum invasion distance (mean \pm s.e.m.). (b) TFM quantified traction stresses exerted by cells on 5kPa 2D polyacrylamide substrate mimicking mammary tumor stiffness by measuring displacement of embedded fluorescent polystyrene beads. Phase image followed by fluorescent images of bead field under stressed and unstressed (post-trypsinization) conditions. Bead displacement yields magnitude ( $|\mathrm{T}|$ ) and distribution of traction stresses ${ }^{42}$. (i) Schematic. (ii) Tension maps (Left); phase images (Right); MCF-10A (Top); MDA-MB-231 (Bottom). Scale bars: $50 \mu \mathrm{m}$. (iii) Force magnitudes exerted by cells at different surface laminin concentrations (mean \pm s.e.m.). (c) Microprinted Covalent HA array. (i) Schematic. (ii) CD44 expressing MCF-10A and MDA-MB-231 cells (red) attached to FL-HA micro-patterned substrates (green) after 24h culture. Scale bars: $50 \mu \mathrm{m}$. (iii) Cells attached to HA squares (mean \pm s.e.m.). (d) Cells rolling on E-selectin surfaces. (i) Schematic. (ii) Phase images. Scale bars: $50 \mu \mathrm{m}$. (iii) Rolling velocities and numbers of MCF-10A cells captured on surface under physiological wall shear stresses (mean \pm s.e.m.). (e) (i) SEM reveals distinct ECM structures deposited by MCF-10A (Left; scale bar: $4 \mu \mathrm{m}$ ) compared to MDA-MB-231 (Right; Scale bar: $5 \mu \mathrm{m}$ ). (ii) IF imaging of cells stained with fluorescein-tagged HA demonstrate expression of HA in both MCF-10A and MDA-MB-231. Scale bars: $50 \mu \mathrm{m}$. (iii) Flow cytometry shows higher CD44 expression in MDA-MB-231 compared to MCF-10A (left: histogram; right: quantified MFI). (f) AFM probe aligned with confocal fluorescence lifetime microscope scans points of interest over cytoplasm, nuclei, and nucleoli. Force-indentation curves used to calculate elastic moduli. (i) Schematic. (ii) Curves (middle) and corresponding images (top, bottom). Scale bars: $10 \mu \mathrm{m}$ (top); $2 \mu \mathrm{m}$ (middle, horizontal), $0.2 \mathrm{nN}$ (middle, vertical); $4 \mu \mathrm{m}$ (bottom). (iii) Depth-dependent elastic moduli (mean \pm s.e.m.). (g) Fluorescent nanoparticles injected into cells and trajectory monitored over time. (i) Schematic. (ii) Cell monitored in real time. Inset: nanoparticle trajectory. Scale bars: $10 \mu \mathrm{m}$ (main); $0.2 \mu \mathrm{m}$ (inset). (iii) MSD values over cumulative time (mean \pm s.e.m.). All $p$-values indicated by Michelin guide scale $\left(p \leq 0.001:[* * *] ; 0.001<p \leq 0.01:[* *] ; 0.01<p \leq 0.05:\left[{ }^{*}\right]\right.$; $0.05<p:$ ns). 
viscoelastic material) $)^{25,26}$. Fluorescently labeled, $100 \mathrm{~nm}$ diameter microspheres were ballistically injected into the cytoplasm and, after overnight incubation, their random intracellular displacement was followed over time (Fig. 2g). Mean squared displacement (MSD) of the microspheres was obtained from the $20 \mathrm{~s}$ trajectory of each microsphere with $30 \mathrm{~ms}$ temporal resolution. Consistent with the above AFM results, the ensemble-averaged MSD of microspheres in MDA-MB-231 cells was greater than that of MCF-10A cells, indicating that the MDA-MB-231 cytoskeleton was substantially softer than that of MCF-10A cells.

Comparative cancer cell stress response and survival. Preferential survival under stressful conditions is a characteristic of metastatic cancer cells; therefore, the two model cell lines were subjected to external stresses characteristic of the tumor microenvironment, such as hypoxia (in $2 \mathrm{D}$ and $3 \mathrm{D}$ ) and low $\mathrm{pH}$. Cell viability, recovery, oxygen consumption (of single cells and populations), and expression of the surface biomarker carcinoembryonic antigen (CEA) were measured.

Hypoxia. Within the primary tumor, oxygen availability can vary dramatically due to location within the tumor and its vascularity; oxygen availability also varies temporally (e.g., due to clots). The effects of hypoxia on cell viability, growth and recovery, and oxygen consumption were tested in a $2 \mathrm{D}$ environment as well as in a pathologically relevant $3 \mathrm{D}$ culture environment. In a hypoxic $\left(1 \% \mathrm{O}_{2}\right) 2 \mathrm{D}$ environment, populations of both cell lines experienced relatively small decreases in viability over a span of three days (Fig. 3a, top). In a 3D culture environment, viability of both cell lines was apparently more sensitive to hypoxia (Fig. 3a, bottom). The normalized MCF-10A cell viability under ambient and hypoxic conditions at day 6 were $100( \pm 20) \%$ and $40( \pm 10) \%$, respectively. The normalized MDA-MB-231 cell viability under ambient and hypoxic conditions at day 6 were $150( \pm 30) \%$ and $76( \pm 20) \%$, respectively. The apparent differences of viability in hypoxic $2 \mathrm{D}$ and $3 \mathrm{D}$ environments require further study but may involve oxygen permeability (surface $v s$. bulk effects in $3 \mathrm{D}$ ), dead cells remaining caught in $3 \mathrm{D}$ scaffolding, or changes in cell adhesion. $\mathrm{O}_{2}$ media concentrations were also measured in the 3D cultures and the normalized bulk oxygen consumption rate at day 6 was determined for both cell lines (Fig. 3b, left). Under ambient conditions, MCF-10A and MDA-MB-231 cells exhibited similar $\mathrm{O}_{2}$ consumption rates; however, their response to hypoxic $\left(1 \% \mathrm{O}_{2}\right)$ treatment differed with no changes in MCF-10A $\mathrm{O}_{2}$ consumption, whereas MDA-MB-231 cells dramatically reduced $\mathrm{O}_{2}$ consumption (four-fold) when cultured in hypoxia (Fig. 3b, left). The observed reduction of $\mathrm{O}_{2}$ consumption by MDA-MB-231 cells may be due to an increased plasticity or adaptability related to their tumorigenic potential. An alternative explanation may be the preexistence of variant MDA-MB-231 cells in the population with reduced $\mathrm{O}_{2}$ metabolism that are positively selected for under hypoxic stress. To examine these possibilities, oxygen consumption rates of single cells isolated in hermetically sealed chambers were measured in normoxic $\left(17 \% \mathrm{O}_{2}\right)$ conditions (Fig. 3b, right). In these measurements, MDA-MB-231 cells had a lower mean OCR but comparable heterogeneity (mean: $2.1 \mathrm{fmol} / \mathrm{min}, \mathrm{CV}: 0.72$ ) than the MCF-10A cells (mean: $4.1 \mathrm{fmol} / \mathrm{min}, \mathrm{CV}: 0.82$ ). The histogram suggests a glycolytic subpopulation of the metastatic cells may become dominant in hypoxic conditions, but further studies are required. This hypothesis is sketched out in Fig. 3c. In addition, measurements of the expression of selected cell surface proteins thought to be involved in metastasis, such as CEA (Fig. 3d), PCLP, and CD44 (Suppl. Fig. 2d), as a function of a normal or hypoxic environment showed that MDA-MB-231 cells increased expression of CEA in response to hypoxic conditions. Future investigations may clarify the effect of hypoxic conditions on the expression of these proteins.
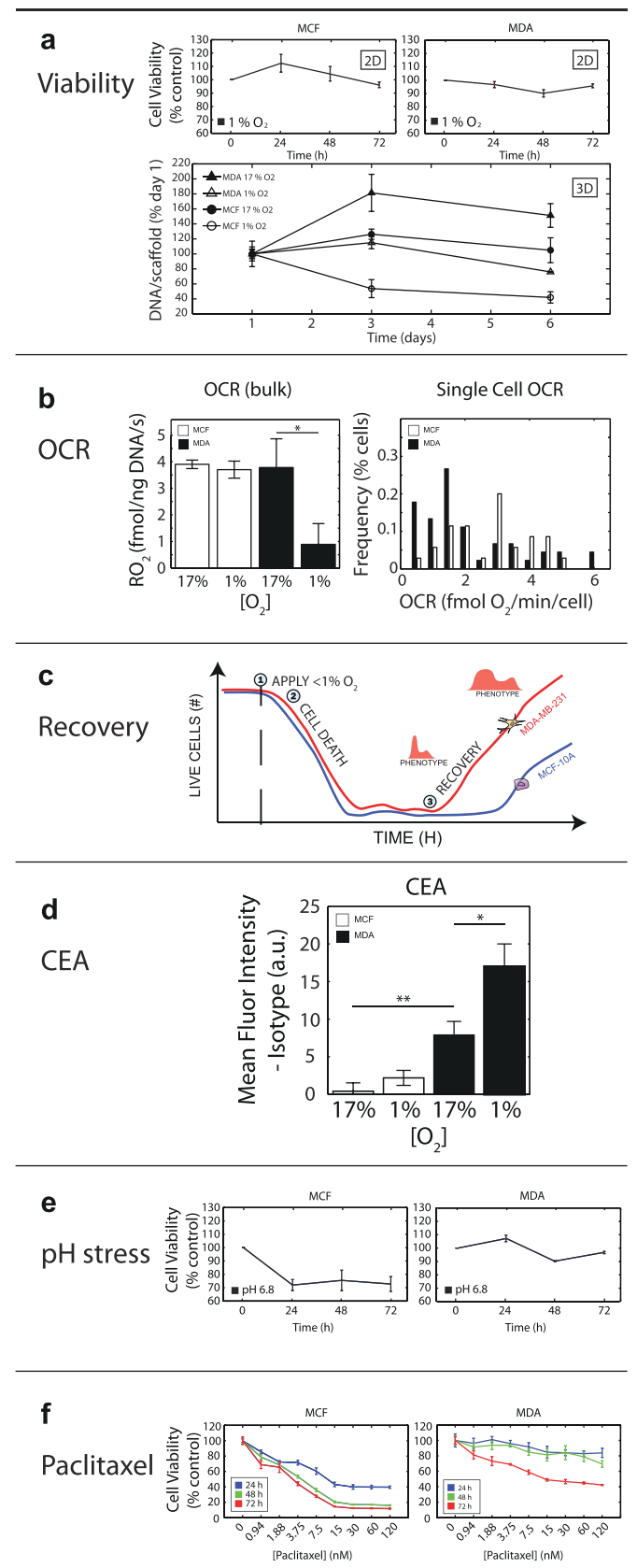

Figure 3 Comparative cell stress responses. (a) Viability under hypoxia in 2D and 3D. Top: (2D) Cells grown in wells (triplicate) for 3 days in $1 \% \mathrm{O}_{2}$. Viability was determined every $24 \mathrm{~h}$ and imaged with an inverted microscope. Cell viability (mean \pm s.e.m.) normalized to day 1 samples. Bottom: (3D) DNA content per scaffold (normalized to day 1 samples) over 6 days growth in normoxic $\left(17 \% \mathrm{O}_{2}\right)$ and hypoxic $\left(1 \% \mathrm{O}_{2}\right)$ conditions in $3 \mathrm{D}$ culture (alginate discs). (b) Oxygen consumption rates. Left: bulk OCR (normalized to DNA content, mean \pm s.e.m.) of cells after 6 days growth in normoxic $\left(17 \% \mathrm{O}_{2}\right)$ or hypoxic $\left(1 \% \mathrm{O}_{2}\right)$ conditions in $3 \mathrm{D}$ culture (alginate discs); Right: histogram of OCR of single cells measured in hermetically sealed chambers $\left(17 \% \mathrm{O}_{2}\right)$. (c) Schematic of a hypothetical model in which phenotypic diversity of MDA-MB-231 cells is relatively enhanced with respect to MCF-10A due to enhanced population recovery after hypoxia-induced cell death. (d) Carcinoembryonic antigen (CEA). Mean fluorescence intensity minus isotype (MFI) of cells grown in $17 \%$ or $1 \% \mathrm{O}_{2}$ (mean \pm s.e.m.). (e) $\mathrm{pH}$-induced stress. Percentages of viable cells grown in media with $\mathrm{pH} 6.8$ (mean \pm s.e.m.). (f) Paclitaxel-induced stress. Percentages of viable cells after 24,48 , and $72 \mathrm{~h}$ incubation with various concentrations of paclitaxel (mean \pm s.e.m.). All $p$-values are indicated according to the Michelin guide scale ( $p \leq 0.001:[* * *] ; 0.001<p \leq 0.01:[* *] ; 0.01<p \leq 0.05:[*] ; 0.05<p$ : ns). 
Chemical stress. Changes in the interstitial chemistry of the microenvironment can also have a profound impact on cancer cell response and metastasis. Low interstitial $\mathrm{pH}$ is typical in the tumor microenvironment due to increased lactic acid secondary to anaerobiosis within anoxic tumors. When cells were grown under acidic conditions, $\mathrm{pH} 6.8$ vs. neutral $\mathrm{pH} 7.4$, and cell proliferation was monitored at fixed time points (microscopic imaging of calcein$\mathrm{AM}$ and ethidium homodimer-1 staining every $24 \mathrm{~h}$ for $72 \mathrm{~h}$ ), MCF-10A viability dropped to $70 \%$ of control by $24 \mathrm{~h}$ and remained there through $72 \mathrm{~h}$ (Fig. 3e), whereas viability of the metastatic cell line was not significantly affected. Additionally, viability of the cell lines was measured after $24 \mathrm{~h}, 48 \mathrm{~h}$, and $72 \mathrm{~h}$ exposure to a range of doses of the mitotic inhibitor paclitaxel (Fig. 3f). Although a clear dose-dependent decrease in viability was observed in both cell lines, the MDA-MB-231 cells were considerably less sensitive to the drug than the MCF-10A cells.

Molecular network signatures for morphology, motility, and stress. The goals of our studies were to broaden the set of features that differentiate between metastatic and non-tumorigenic cells to include physical/mechanical properties and to generate hypotheses about how molecular-scale factors (e.g., protein and transcript level changes) impact or are impacted by these properties. The following molecular network analysis attempts to identify putative molecular origins of the data from Fig. 1 and Fig. 2. Notably, in addition to the transcriptomic data, proteomics datasets from each cell line were collected both unperturbed and perturbed by various antagonists that could potentially alter parameters such as stress response (e.g. paclitaxel) and motility and morphology (ROCK Inhibitor Y-27632). This allowed us to further refine our estimates of definition of genes from the network that were putatively connected to particular phenotypic characteristics. To connect the molecular and the biophysical, we proceeded in three steps: 1) we identified relevant transcriptome data (224 experimental conditions from the Gene Expression Omnibus) for the two cell lines and generated quantitative differential proteomics data (see Methods); 2) we derived a computational model of cellular regulation; and 3) we identified subsets of that model that are likely related to physical properties of the cells.

Our transcriptional regulatory network model (Suppl. Fig. 3) contains 1866 genes, 220 of which encode transcription factors (TFs) and highlights factors that differentiate between metastatic and premalignant cell types (see Methods). In this model, each gene is represented as a 'node'. If a gene's abundance is regulated by another gene, this is denoted with an 'edge' between those genes. In Fig. 4a, we show the transcription factor subset. Yellow nodes or edges indicate specificity to MCF-10A cells, whereas blue nodes or edges are more specific to MDA-MB-231 cells. This network shows a substantial bias towards MDA-MB-231 specific nodes and edges.

We interrogated our derived total network (Suppl. Fig. 3) to identify sub-networks whose regulation was both highly differentiated between cell types and likely connected to physical cell properties (morphology, motility, and stress). Proteomics measurements were overlaid onto these networks.

In Figs. $4 \mathrm{~b}-\mathrm{d}$, we show some of the 1-hop sub-networks (i.e., one degree of separation between genes) selected from the millions of 1hop sub-networks identified. These particular networks contain one or more interesting features: connections are specific to one cell line; the genes are of interest to the PS-OC community and/or implicated in cell biophysical properties; and/or the genes correspond to differentiating proteomics data.

As noted above, there were numerous morphologic differences between our cells, including factors like width-to-length ratio and nuclear disorder factors. Notably, for the morphology sub-network (Fig. 4b), we observe strong connections among FBN1 (encoding fibrillin, a major extracellular microfibril in connective tissues),
ZEB1 (encoding a zinc finger transcription factor that represses Tlymphocyte-specific IL2 gene), and TWIST1 (encoding a basic helixloop-helix transcription factor). The inferred network connects these genes, whose products are known to impact the morphology of cells. $F B N 1$ is of particular interest because it has a large number of MDA-MB-231-specific edges. It is a connective protein that provides structural support for surrounding tissue. The large number of specific edges suggests that FBN1 plays a role in the deformability of the MDA-MB-231 cells. The network analysis further infers that FBN1 has regulatory interactions with gene products involved in cellular differentiation, adhesion, structure, and integrity, such as TGF $\beta 3$ (encoding transforming growth factor $\beta 3$ ), $M M P 2$ (encoding matrix metalloproteinase-2), LOX (encoding lysyl oxidase), and ACTA2 (encoding smooth muscle aortic $\alpha$-actin). TGF $\beta$-3, MMP-2, and LOX have previously been shown to be involved in tumor growth and metastasis ${ }^{27,28}$, whereas to our knowledge ACTA2 has not.

An analysis of motility network signatures in MCF-10A and MDA-MB-231 cells identified ITG $\beta 4$ (encoding integrin $\beta 4$ ) as a central node (Fig. 4c). Integrin $\beta 4$ is a member of the integrin family necessary for cell-matrix and cell-cell adhesion and for motility ${ }^{29,30}$. Our model suggests that it is a potential regulator of many gene products involved in adhesion, migration, and invasion. This particular network also contains a large number of differentially abundant gene products based on proteomics, most notably SERPINB5 (encoding serpin peptidase inhibitor, clade B, member 5), which is upregulated in the non-malignant MCF-10A cells and is known to act as a tumor suppressor and block the metastatic properties of mammary tumors, further suggesting a role in differential regulation.

In the survival network, we focused initially on HIF1 $\alpha$ (hypoxiainducible factor-1 $\alpha$ ), as it encodes a transcription factor known to be involved in cell response to hypoxia ${ }^{31}$. We focused on this gene because it is over-expressed in the metastatic cells able to survive in areas of low $\mathrm{O}_{2}$ within a tumor (Fig. 4d). Products of genes in this regulatory network ultimately affect the survival of a cell through proliferation or apoptosis. Our model suggests that $c-$ Met has regulatory relationships with both HIFl $\alpha$ and ITG $\beta 4$ in the transcription factor regulatory network, consistent with the reports from Schelter et al. ${ }^{32}$ and Giancotti ${ }^{33}$ respectively. In addition, we suggest that HIF1 $\alpha$ regulates $L O X$, which has been shown to be critical for hypoxia-induced metastasis ${ }^{34-36}$.

\section{Discussion}

This trans PS-OC laboratory network study demonstrates that cell lines can be cultured with genotypic and phenotypic uniformity across geographically and technologically disparate institutions to be used in comparative studies. The results from each laboratory can be pooled with confidence, given the known uniformity in cell maintenance and thus the phenotypic and genotypic make-up of the cells. This is in contrast to typical comparisons with results from literature that are fraught with the pitfalls inherent in the use of different experimental conditions and materials. In the present work, the physical differences between a non-tumorigenic cell line (MCF10A) and a metastatic cell line (MDA-MB-231) that are both extensively used in research were characterized. The integrated results generate a composite and multifaceted picture (detailed in Table 2) that is more complete than one or a few experimental approaches would have provided. In proposing to develop a laboratory network approach to study the physical properties of cancer cells, there was concern about the inherent mutator genotype of the cell lines as well as phenotypic plasticity due to variant culturing conditions, which could result in differing cell lineages and phenotypes potentially confounding interpretation and integration of results across the twelve PS-OCs. Consequently, considerable work was done to ensure that the two cell lines, culture media, and growth conditions were standardized and that cell growth and overall morphology were documented at regular intervals for quality control. Subsequently, 

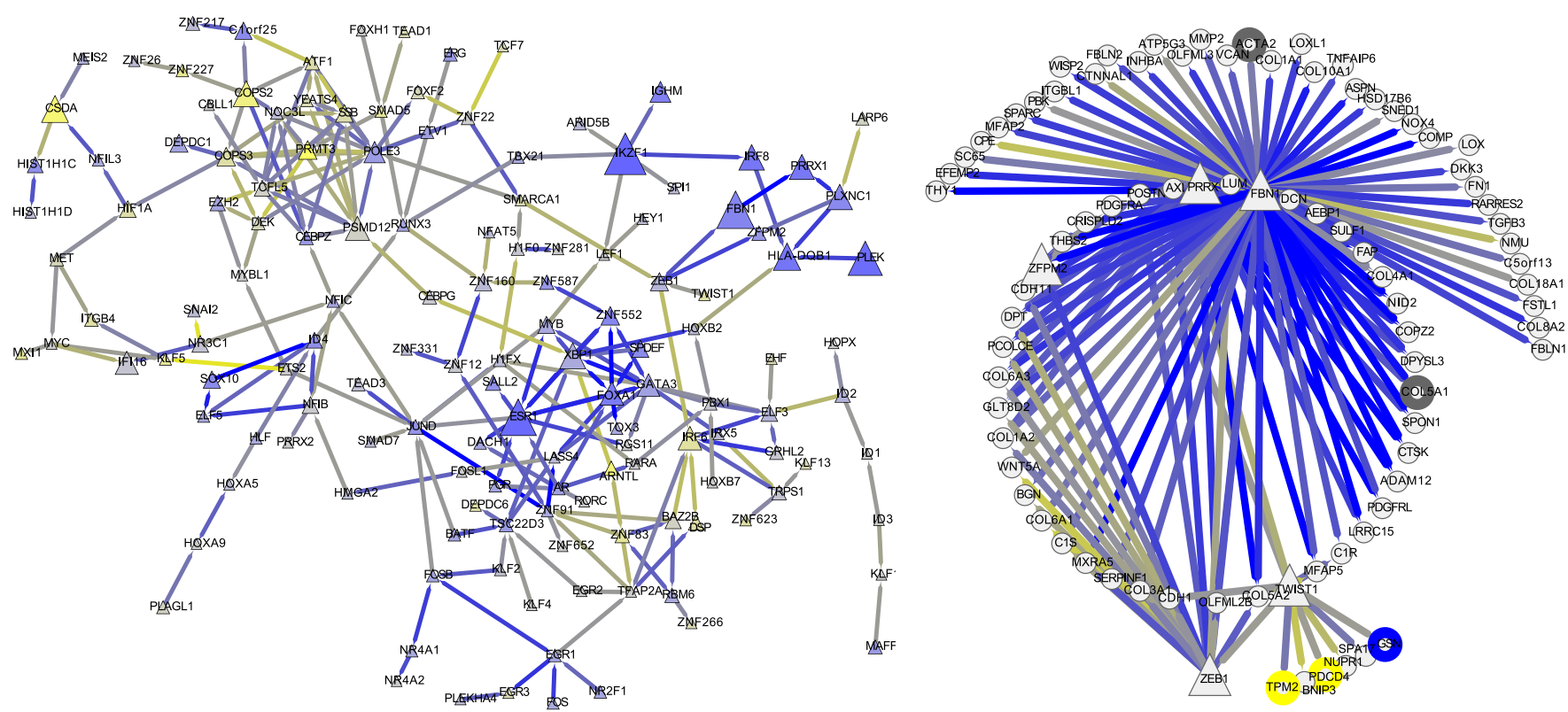

C

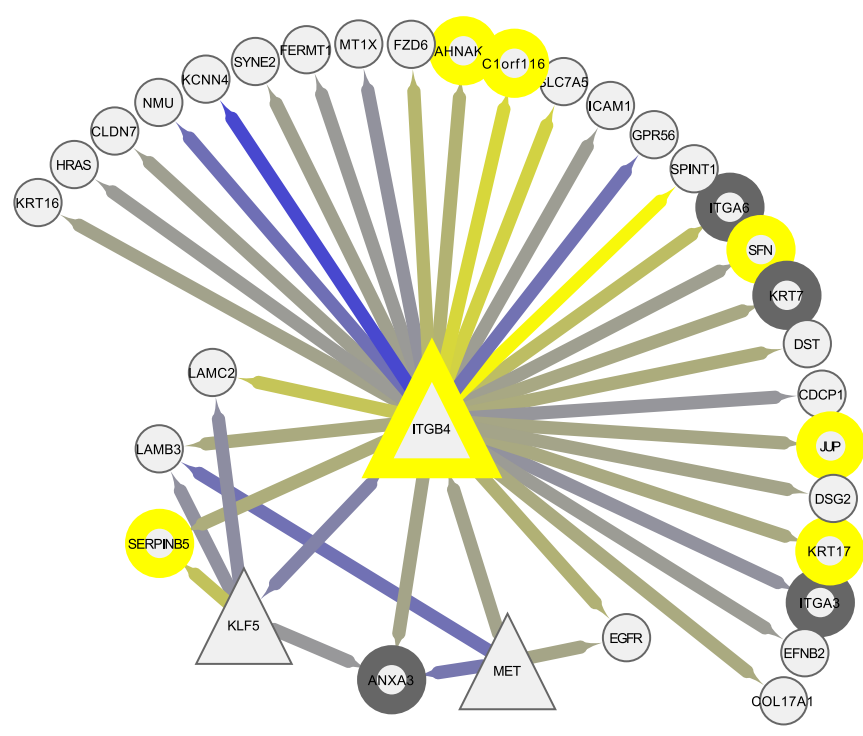

d

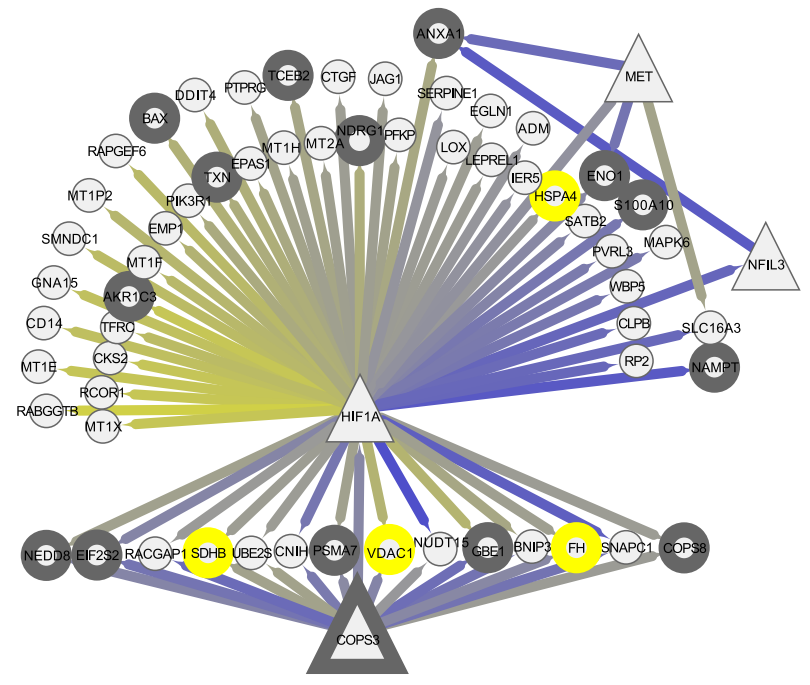

Figure 4 Comparative molecular signatures for morphology, motility, and stress. (a) The largest connected subnetwork of transcription factors from the master network (Suppl. Fig. 3) with nodes colored to provide a "summary" of the entire network. Node size shows the number of edges (connecting lines) in the master network that were above a cutoff for specificity to either cell line. Larger nodes have more cell-line-specific edges; the largest, IKZF1, has 67 edges above the threshold. Node color is determined by the ratio of above-cutoff edges specific to MCF-10A vs. MDA-MB-231, with yellow denoting more MCF-10A edges and blue more MDA-MB-231 edges. Nodes with many edges specific to one cell line or the other are therefore large and brightly colored, such as IKZF1 or COPS2. (b-d) One-hop networks from transcription factor regulators $(\triangle)$ to their targets $(\bigcirc)$. Each gene is represented as a 'node'. If a gene's abundance is regulated by another gene, this is denoted with an 'edge' between those genes. Color of an edge indicates the specificity of that regulatory relationship to either MCF-10A cells (yellow) or MDA-MB-231 cells (blue). Relationships that are equally present in both cell types are demarked grey. Node border color indicates differential proteomics results. Yellow border nodes are upregulated in MCF-10A cells. Blue border nodes are upregulated in MDA-MB-231 cells. Grey bordered nodes were quantified and found to be equivalent in both cell types. (b) Morphology network. The 1-hop morphology network from FBN1 and TWIST1, LOX and LOXL1, both putatively regulated by FBN1. Both FBN1 and TWIST1 are putatively regulated by ZEB1. Also shown are the large number of MDA-MB-231 edges from FBN1 and a fairly even distribution of edges from $Z E B 1$. (c) Motility network. The 1-hop network from ITGB4. ITGB4 is itself a gene of interest and is inferred to regulate EGFR and several laminins. (d) Stress response network. The 1-hop network from HIF1A, a transcription factor and gene of interest. It is putatively regulated by MET (upper triangle), which is also inferred to regulate ITGB4. HIF1A putatively regulates two more genes of interest, LOX (also a putative target of FBN1 and SATB2).

each lab employed their own unique procedures for cell characterization as provided in Table 1 and the supplemental information.

Integration of the results across the study highlights the similarities and differences between the cell types. For example, the apparent phenotypic plasticity of the metastatic MDA-MB-231 cells compared with the non-tumorigenic MCF-10A cells is illustrated at the $20 \mathrm{~nm}$ scale, at which an increase in nuclear disorder strength indicative of more variable nuclear heterochromatin structure was seen in 
MDA-MB-231 cells, and at the $\mu \mathrm{m}$ scale, at which the cellular deformability of the metastatic cells was found to be four-fold more elastic than the non-tumorigenic cells as measured by AFM (Table 2). These results are consistent with the role of the cytoskeleton in malignancy. The cytoskeleton functions not only as the cell backbone but also plays an active role in cell division, differentiation and signal transduction; modifications in the cytoskeleton have been associated with early carcinogenesis ${ }^{37}$. In addition, the nuclear disorder strength is currently under evaluation as a potential early diagnostic marker of metastasis ${ }^{38}$ and thus may be an indicator of early events in carcinogenesis. Measured values for $L_{\mathrm{d}}$ have been found to be elevated with higher neoplastic behavior in genetically altered but microscopically indistinguishable human colon cancer cell lines ${ }^{12}$. One benefit of this kind of trans-network study is the ability to look at relationships between observations. For example, the AFM data presented suggests that metastatic cells are softer. Interestingly, the TFM data suggests that metastatic cells are able to exert increased force. This compound observation is both phenotypically intuitive and surprising.

One of the unanticipated findings was that motility of metastatic MDA-MB-231 cells is profoundly different than that of MCF-10A cells. MDA-MB-231 cells, in spite of exhibiting a slower overall speed, traveled farther and more linearly in the more pathologically relevant 3D motility assay than MCF-10A cells, which move in a circular manner. Previously, motility measurements for these cells were performed in a 2D environment; in this milieu, MCF-10A cells traveled farther than MDA-MB-231 cells. Motility was further explored through measurements of adhesion and rolling on matrices with different surface ligands. The MDA-MB-231 cell surface is very different from that of MCF-10A cells, and these differences impact interactions with the microenvironment. CD44 distribution on the two cell lines differs; MCF-10A cells exhibit abundant CD44 at the cell-substrate interface, whereas the MDA-MB-231 cells had less detectable CD44 at the cell-substrate interface. In addition, lipid rafts were detectable on the metastatic cells but not on the MCF-10A cells. These results suggest that although the general topography of the two cell types is similar, the surface presentation of the cell adhesion protein CD44 and formation of lipid raft domains containing additional cell surface receptors differ. These results are consistent with previous findings of elevated levels of cholesterol-rich lipid rafts in breast and prostate cancer cells compared with their normal counterparts $^{13,22}$.

The metastatic cells exhibited less adhesion and rolling on E-selectin and HA surfaces, consistent with the lower expression of CD44 detected at the substrate/membrane contact areas in TIRF images of the cells. The labeling experiments support the idea that the general topographies of the two cell lines are similar. The exclusion of CD44 from the near-surface contact area in MDA-MB-231 cells or lack of proper post-translational CD44 modification may explain their lack of binding to the HA micro-patterned surface. These results differed substantially from those of Afify et al. ${ }^{39}$ who found that CD44 was required for breast cancer cell adhesion since anti-CD44 antibodies disrupted cell adhesion to HA-coated wells. In their study, Afify et al. only investigated the adhesion of two breast cancer cells lines: an MDA-MB-468 cell line that expresses high levels of CD44, and a T47D cell line with low to negligible expression of CD44;

Table 2 | Comparative experimental physical parameters for non-tumorigenic and metastatic cells

$\begin{array}{lll}\text { Cell Physical Parameter Non-tumorigenic MCF-10A Metastatic MDA-MB-231 } & \text { Nat }\end{array}$

\section{Morphology}

Cell shape and volume

$\left(2 D^{a}, 3 D \text {, and nuclear shape }\right)^{b}$

Nuclear architecture

Effect of matrix stiffness on cell proliferation and morphology

Cell surface (CD44 expression patterns and lipid raft distribution)
- Smaller cell volume, spherical cell shape

- Heterogeneous, non-spherical nuclear shape

Baseline

Sensitive (increased proliferation with increased stiffness)

- Abundant CD44 at cell surface-substrate interface

- No measurable lipid rafts
- Larger cell width/length ratio and volume

- Kidney bean, non-spherical nuclear shape $80 \%$ increased nuclear disorder Insensitive

- Reduced CD44 at cell surface-substrate interface

- Detectable lipid rafts

\section{Motility}

1D motility in fibronectin channel

2D motility on collagen

3D motility in collagen

Traction force on laminin

Rolling on E-selectin

Adhesion on hyaluronic acid

Less displacement
More displacement
Less displacement (circular motion)
Less traction force
Yes
Yes

Less displacement

More displacement

Less traction force

Yes

More displacement
Less displacement
More displacement (linear motion)
More traction force
No
No

\section{Mechanics}

Deformability, elasticity (AFM

nanoindentation)

Internal fluidity (microparticle displacement) Less fluidity

\section{Stress response and survival}

Hypoxia

Low $\mathrm{pH}$ environment

Paclitaxel
Less deformable, less elastic

More deformable, more elastic

More fluidity

${ }^{a}$ Abbreviations used: 1D: one-dimensional; 2D: 2-dimensionional; 3D: 3-dimensionional; CEA: carcinoembryonic antigen.

bee text for additional explanation of experimental results.

- Low cell viability, slow population recovery

- No significant change in $\mathrm{O}_{2}$ consumption

- Low nominal CEA expression with small increase

Greater toxicity

Greater sensitivity
- Low cell viability, subpopulation survival with fast recovery and greater proliferation

- Fourfold reduction in $\mathrm{O}_{2}$ consumption

- Low nominal CEA expression with moderate

increase

Sensitive

Sensitive 
MDA-MB-231 cells were shown to express CD44, but at a lower level, and their adhesion was not examined. In the Afify et al. analysis, HA was presented singularly, whereas in our studies HA was presented adjacent to PEG-ylated regions. Some of the present authors have previously shown that adhesion preference to HA-presenting region depends on the presentation of adjacent molecules ${ }^{40}$.

When subjected to stressors such as low $\mathrm{O}_{2}$ and acidosis, both MDA-MB-231 and MCF-10A cells exhibited reduced viability as compared with culture under ambient $\mathrm{O}_{2}$ and physiological $\mathrm{pH}$; MDA-MB-231 cell numbers were maintained to a greater degree than MCF-10A, illustrative of the metabolic flexibility exhibited by metastatic cells compared to the non-tumorigenic cell line. MCF$10 \mathrm{~A}$ cells tolerated low $\mathrm{O}_{2}$ better in $2 \mathrm{D}$ as compared with $3 \mathrm{D}$ culture, with total cell numbers dropping by more than $50 \%$ after six days of culture in 3D. This finding was unexpected, and may point to different mechanisms of stress response mediated by culture dimensionality. In hypoxic conditions, the four-fold reduction in $\mathrm{O}_{2}$ consumption by the 3D-cultured MDA-MB-231 cells, in contrast to the unchanged MCF-10A consumption, may be due either to increased phenotypic plasticity or to the preexistence of an emergent subpopulation with reduced $\mathrm{O}_{2}$ metabolism that survive under hypoxic stress. These results demonstrate that it is important to monitor not only the whole cell population but also single cells. The single cell studies of oxygen consumption support the idea that a subset of MDA-MB-231 cells restored the population after these microenvironmental stress-caused evolutionary bottlenecksa hypothesis that is also consistent with the results of the viability studies. Taken together, these results confirm and extend prior observations of the inherent capacity of metastatic cells to respond to microenvironment stressors of low $\mathrm{O} 2$ partial pressure and acidic $\mathrm{pH}$.

Proteomic network analysis of MCF-10A and MDA-MB-231 cells confirmed changes in cell morphology and motility and identified genes that are involved in multiple metastatic hallmark features. For example, LOX, which is regulated in the morphology and survival networks by $F B N 1$ and $H I F 1 \alpha$, respectively, is involved in extracellular matrix remodeling and is a hypoxia-responsive gene that represses Ecadherin, leading to cellular transformation and invasion. Through a combination of regulatory network inferences and proteomic studies, we interrogated the molecular origins of several classic phenotypic "hallmark" properties of tumor progression and metastasis.

Clearly, an important next step is to bring the biophysical assays presented in this paper to the in vivo realm. Some of these assays could be modified and readily extended to the in vivo case, such as quantitative single-cell migration, traction force microscopy, and particle tracking microrheology. Other measurements cannot be readily conducted in vivo, such as $3 \mathrm{D}$ cytometry.

In sum, the results of experiments comparing parameters of cell lines chosen to model the non-tumorigenic and metastatic states generated internally consistent results. The metastatic cells were more physically flexible and viable under a wider range of stresses than the non-tumorigenic cells. In addition, cell surface protein expression was related to the motility parameters measured. This pilot study validates the laboratory network approach and the use of physical sciences techniques to investigate cancer cell biology.

\section{Methods}

Cell lines. Cells were grown with standardized media and culture conditions described by Guise et al. ${ }^{41}$ for MCF-10A cells and Debnath et al. ${ }^{4}$ for MDA-MB-231 cells. Additional specific cell growth conditions for specific assays are provided in the supplementary information (SI Methods).

Cell morphology assays. Specific assay conditions are provided in SI Methods.

Cell motility and mechanics assays. Specific assay conditions are provided in SI Methods.

Cell stress response and survival assays. Specific assay conditions are provided in SI Methods.
Gene and protein expression and analysis. Specific assay conditions are provided in SI Methods.

1. Wirtz, D., Konstantopoulos, K. \& Searson, P. C. The physics of cancer: the role of physical interactions and mechanical forces in metastasis. Nature reviews. Cancer 11, 512-522 (2011)

2. Hanahan, D. \& Weinberg, R. A. Hallmarks of cancer: the next generation. Cell 144, 646-674 (2011).

3. Davies, P. C., Demetrius, L. \& Tuszynski, J. A. Cancer as a dynamical phase transition. Theor Biol Med Model 8, 30 (2011).

4. Debnath, J., Muthuswamy, S. K. \& Brugge, J. S. Morphogenesis and oncogenesis of MCF-10A mammary epithelial acini grown in three-dimensional basement membrane cultures. Methods 30, 256-268 (2003).

5. Cailleau, R., Mackay, B., Young, R. K. \& Reeves, W. J., Jr. Tissue culture studies on pleural effusions from breast carcinoma patients. Cancer research 34, 801-809 (1974).

6. Subik, K. et al. The Expression Patterns of ER, PR, HER2, CK5/6, EGFR, Ki-67 and AR by Immunohistochemical Analysis in Breast Cancer Cell Lines. Breast cancer : basic and clinical research 4, 35-41 (2010).

7. Akkiprik, M. et al. Dissection of signaling pathways in fourteen breast cancer cell lines using reverse-phase protein lysate microarray. Technology in cancer research \& treatment 5, 543-551 (2006).

8. Prvulovic, I., Kardum-Skelin, I., Sustercic, D., Jakic-Razumovic, J. \& Manojlovic, S. Morphometry of tumor cells in different grades and types of breast cancer. Collegium antropologicum 34, 99-103 (2010).

9. Nafe, R., Franz, K., Schlote, W. \& Schneider, B. Morphology of tumor cell nuclei is significantly related with survival time of patients with glioblastomas. Clinical cancer research : an official journal of the American Association for Cancer Research 11, 2141-2148 (2005).

10. Kenny, P. A. et al. The morphologies of breast cancer cell lines in threedimensional assays correlate with their profiles of gene expression. Molecular oncology 1, 84-96 (2007).

11. Nandakumar, V., Kelbauskas, L., Johnson, R. \& Meldrum, D. Quantitative characterization of preneoplastic progression using single-cell computed tomography and three-dimensional karyometry. Cytometry. Part A : the journal of the International Society for Analytical Cytology 79, 25-34(2011).

12. Damania, D. et al. Role of cytoskeleton in controlling the disorder strength of cellular nanoscale architecture. Biophysical journal 99, 989-996 (2010).

13. Butcher, D. T., Alliston, T. \& Weaver, V. M. A tense situation: forcing tumour progression. Nature reviews. Cancer 9, 108-122 (2009).

14. Yeung, T. et al. Effects of substrate stiffness on cell morphology, cytoskeletal structure, and adhesion. Cell motility and the cytoskeleton 60, 24-34 (2005).

15. Shain, K. H., Landowski, T. H. \& Dalton, W. S. The tumor microenvironment as a determinant of cancer cell survival: a possible mechanism for de novo drug resistance. Current Opinion in Oncology 12, 557-563 (2000).

16. Sneath, R. J. \& Mangham, D. C. The normal structure and function of CD44 and its role in neoplasia. Molecular pathology : MP 51, 191-200 (1998).

17. Brown, R. L. et al. CD44 splice isoform switching in human and mouse epithelium is essential for epithelial-mesenchymal transition and breast cancer progression. The Journal of clinical investigation 121, 1064-1074 (2011).

18. Zen, K. et al. CD44v4 is a major E-selectin ligand that mediates breast cancer cell transendothelial migration. PloS one 3, e1826 (2008).

19. Fraley, S. I. et al. A distinctive role for focal adhesion proteins in three-dimensional cell motility. Nature cell biology 12, 598-604 (2010).

20. Fraley, S. I., Feng, Y., Giri, A., Longmore, G. D. \& Wirtz, D. Dimensional and temporal controls of three-dimensional cell migration by zyxin and binding partners. Nat Commun 3, 719 (2012).

21. Balzer, E. M. et al. Physical confinement alters tumor cell adhesion and migration phenotypes. FASEB J 26, 4045-4056 (2012).

22. Provenzano, P. P. et al. Collagen density promotes mammary tumor initiation and progression. BMC medicine 6, 11(2008).

23. Bresalier, R. S. et al. Enhanced sialylation of mucin-associated carbohydrate structures in human colon cancer metastasis. Gastroenterology 110, 1354-1367 (1996).

24. Lee, M. H. et al. Mismatch in mechanical and adhesive properties induces pulsating cancer cell migration in epithelial monolayer. Biophys J 102, 2731-2741 (2012).

25. Wirtz, D. Particle-Tracking Microrheology of Living Cells: Principles and Applications. Annual Review of Biophysics 38, 301-326 (2009).

26. Wu, P.-H. et al. High-throughput ballistic injection nanorheology to measure cell mechanics. Nat. Protocols 7, 155-170 (2012)

27. Egeblad, M. \& Werb, Z. New functions for the matrix metalloproteinases in cancer progression. Nature reviews. Cancer 2, 161-174 (2002).

28. Erler, J. T. et al. Lysyl oxidase is essential for hypoxia-induced metastasis. Nature 440, 1222-1226 (2006).

29. Sonnenberg, A. et al. Integrin alpha $6 /$ beta 4 complex is located in hemidesmosomes, suggesting a major role in epidermal cell-basement membrane adhesion. The Journal of cell biology 113, 907-917 (1991).

30. Rüegg, C. et al. Role of integrin alpha 4 beta 7/alpha 4 beta $\mathrm{P}$ in lymphocyte adherence to fibronectin and VCAM-1 and in homotypic cell clustering. The Journal of cell biology 117, 179-189 (1992). 
31. Lee, J. W., Bae, S. H., Jeong, J. W., Kim, S. H. \& Kim, K. W. Hypoxia-inducible factor (HIF-1)alpha: its protein stability and biological functions. Experimental of molecular medicine 36, 1-12 (2004).

32. Dews, M. et al. The Myc-miR-17 similar to 92 Axis Blunts TGF beta Signaling and Production of Multiple TGF beta-Dependent Antiangiogenic Factors. Cancer research 70, 8233-8246 (2010).

33. Giancotti, F. G. Targeting integrin beta 4 for cancer and anti-angiogenic therapy. Trends in pharmacological sciences 28, 506-511 (2007).

34. Erler, J. T. \& Giaccia, A. J. Lysyl oxidase mediates hypoxic control of metastasis. Cancer research 66, 10238-10241 (2006)

35. Erler, J. T. et al. Lysyl oxidase is essential for hypoxia-induced metastasis. Nature 440, 1222-1226 (2006)

36. Erler, J. T. et al. LOX is essential for hypoxia-induced metastasis. Radiotherapy and Oncology 78, S5-S5 (2006).

37. Bernstein, B. W. \& Bamburg, J. R. A proposed mechanism for cell polarization with no external cues. Cell motility and the cytoskeleton 58, 96-103 (2004).

38. Jun Soo, K., Prabhakar, P., Vadim, B. \& Igal, S. The influence of chromosome density variations on the increase in nuclear disorder strength in carcinogenesis. Physical biology 8, 015004 (2011).

39. Afify, A., Purnell, P. \& Nguyen, L. Role of CD44s and CD44v6 on human breast cancer cell adhesion, migration, and invasion. Exp Mol Pathol 86, 95-100 (2009).

40. Dickinson, L. E., Ho, C. C., Wang, G. M., Stebe, K. J. \& Gerecht, S. Functional surfaces for high-resolution analysis of cancer cell interactions on exogenous hyaluronic acid. Biomaterials 31, 5472-5478 (2010).

41. Guise, T. A. Parathyroid hormone-related protein and bone metastases. Cancer 80, 1572-1580 (1997).

42. Paszek, M. J. et al. Tensional homeostasis and the malignant phenotype. Cancer Cell 8, 241-254.

\section{Acknowledgements}

We thank Jack R. Staunton and Denis Wirtz for taking leadership in preparing this manuscript, and we thank Thea D. Tlsty and Barbara L. Hempstead for their input on the choice of cell lines for this project. This work was supported by the following grants from the United States National Cancer Institute: U54CA143862 to P.C.W.D., U54CA143876 to M.L.S., U54CA143798 to F.M., U54CA143970 to R.A.G., U54CA143868 to D.W., U54CA143874 to A.V.O., U54CA143837 to M.F., U54CA143869 to T.V.O., U54CA143803 to R.H.A., U54CA143906 to P.K., U54CA143836 to J.T.L., and U54CA143907 to W.D.H. The content is solely the responsibility of the authors and does not necessarily represent the official views of the National Cancer Institute or the National Institutes of Health.

\section{Author contributions}

The PS-OC Network members listed at the end of the paper all contributed to the PS-OC Cell Line Project. The following PS-OC Network members contributed significantly to the writing of this manuscript and the preparation of figures: Jack R. Staunton and Denis Wirtz. The PS-OC Publication Team conceptualized the paper, the PS-OC Data Analysis Team Leaders analyzed the data and prepared preliminary figures, the PS-OC Data Analysis Team Members conducted experiments and analyzed the data, and the PS-OC Network conducted experiments, analyzed data, and provided scientific input.

Authorship leaders Jack R. Staunton, Denis Wirtz. Atomic force microscopy (Arizona State University PS-OC members) Jack R. Staunton, Alexander Fuhrmann, Robert Ros; Ballistic intracellular nanorheology (Johns Hopkins University PS-OC members) Pei-Hsun Wu, Wei-Chiang Chen, Yiider Tseng, Denis Wirtz; Brightfield and fluorescence microscopy imaging (University of Southern California PS-OC members) Shannon M. Mumenthaler, Nathan C. Choi; Cell line propagation and network-wide distribution (Princeton University PS-OC members at University of California, San Francisco) Philippe Gascard, Chira Chen-Tanyolac, Steve Oh, Luis Estevez-Salmeron, Thea D. Tlsty; Cell surface receptor expression levels (Johns Hopkins University PS-OC members) Matthew Dallas, Konstantinos Konstantopoulos; Computational analysis, network inference, and visualization (University of Southern California PS-OC members at New York University) Christopher S. Poultney, Alex Greenfield, Richard Bonneau; Data sharing platform development (University of Southern California PS-OC member) Carl Kesselman; Differential interference contrast microscopy (The Scripps Research Institute PS-OC members at Oregon Health \& Science University) Kevin G. Philips, Garth W. Tormoen, Owen J.T. McCarty; LC-MS/MS proteomics (University of Southern California PS-OC members) Shannon M. Mumenthaler, Jenny C. Wan, Ahyoung Joo, Jonathan E. Katz, Parag
Mallick; Micro-patterning and extracellular matrix secretion (Johns Hopkins University PS-OC members) Abigail Hielscher, Laura Dickinson, Sharon Gerecht; Nanoparticle delivery (The Methodist Hospital Research Institute PS-OC members) Biana Godin, Srimeenakshi Srinivasan, Jenolyn F. Alexander, Paolo Decuzzi, Wadih Arap, Renata Pasqualini; (W. Arap and R. Pasqualini are at the MD Anderson Cancer Center) One-dimensional cell migration and stress gradients (Princeton PS-OC members) Guillaume Lambert, Liyu Liu, David Liao, Robert H. Austin; Paclitaxel dose curves (University of Southern California PS-OC member) Shannon M. Mumenthaler; Partial wave spectroscopic microscopy (Northwestern University PS-OC members) Dhwanil Damania, Yolanda Stypula, Christine Will, Hariharan Subramanian, John Marko, Vadim Backman; RNA fluorescent in-situ hybridization Kevin Kung, Anna Lyubimova, Alexander van Oudenaarden; Scientific input Joseph E. Aslan; Single-cell oxygen consumption (Arizona State University PS-OC members) Laimonas Kelbauskas, Shashanka Ashili, Patti Senechal, Courtney Hemphill, Deirdre R. Meldrum; Single-cell tomographic imaging and three-dimensional morphometry (Arizona State University PS-OC members) Vivek Nandakumar, Laimonas Kelbauskas, Patti Senechal, Courtney Hemphill, Roger H. Johnson, Deirdre R. Meldrum; Substrate stiffness and growth (University of California, Berkeley PS-OC members at University of California, San Francisco) Christian Frantz, Johnathon N. Lakins, Matthew J. Paszek, Valerie M. Weaver; Two and three-dimensional cell migration (Johns Hopkins University PS-OC members) Stephanie I. Fraley, Denis Wirtz; Three-dimensional hypoxia and oxygen consumption studies (Cornell University PS-OC members) Scott S. Verbridge, Brian Kwee, Claudia Fischbach; Total internal reflection fluorescence microscopy and cell rolling (Cornel University PS-OC members) Yue Geng, Kuldeepsinh Rana, Michael R. King; Traction force microscopy and wound healing assay (Cornell University PS-OC members) Casey M. Kraning-Rush, Cynthia A. Reinhart-King; Viability, $\mathrm{pH}$ and $\mathrm{O}_{2}$ stress (H. Lee Moffitt Cancer Center and Research Institute PS-OC members) Jonathan Wojtkowiak, Veronica Estrella, Arig Ibrahim-Hashim, Mark C. Lloyd, Robert A. Gatenby, Robert J. Gillies; Center Leadership Arizona State University PS-OC: Paul C.W. Davies, William M. Grady; Cornell University PS-OC: Michael L. Shuler, Barbara L. Hempstead; Dana-Farber Cancer Institute PS-OC: Franziska Michor, Eric C. Holland; H. Lee Moffitt Cancer Center and Research Institute PS-OC: Robert A. Gatenby, Robert J. Gillies; Johns Hopkins University PS-OC: Denis Wirtz, Gregg L. Semenza; Massachusetts Institute of Technology PS-OC: Alexander van Oudenaarden, Tyler Jacks; The Methodist Hospital Research Institute PS-OC: Mauro Ferrari, Steven A. Curley; Northwestern University PS-OC: Thomas V. O'Halloran, Jonathan Widom, Jonathan D. Licht; Princeton University PS-OC: Robert H. Austin, Thea D. Tlsty; The Scripps Research Institute PS-OC: Peter Kuhn, Kelly J. Bethel; University of California, Berkeley PS-OC: Jan T. Liphardt, Valerie M. Weaver; University of Southern California PS-OC: W. Daniel Hillis, David B. Agus; PS-OC Data Analysis Team Leaders Owen J.T. McCarty, Cynthia A. Reinhart-King, Sharon Gerecht, Parag Mallick, Roger H. Johnson; PS-OC Data Analysis Team Members Richard Bonneau, Matthew Dallas, Dhwanil Damania, Veronica Estrella, Claudia Fischbach, Jasmine Foo, Stephanie I. Fraley, Christian Frantz, Robert A. Gatenby, Yue Geng, Sharon Gerecht, Biana Godin, Alex Greenfield, Arig Ibrahim-Hashim, Roger H. Johnson, Casey M. Kraning-Rush, Guillaume Lambert, David Liao, Parag Mallick, Owen McCarty, Deirdre Meldrum, Franziska Michor, Shannon M. Mumenthaler, Vivek Nandakumar, Kevin Phillips, Christopher S. Poultney, Jack R. Staunton, Garth Tormoen, Scott S. Verbridge, Jonathan Wojtkowiak, Pei-Hsun Wu; PS-OC Data Integration Leaders Valerie M. Weaver, Denis Wirtz; PS-OC Publication Team Robert H. Austin, Parag Mallick, Owen J.T. McCarty, Thea D. Tlsty, Valerie M. Weaver, Denis Wirtz.

\section{Additional information}

Supplementary information accompanies this paper at http://www.nature.com/ scientificreports

Data: All data files are deposited and viewable at the Physical Sciences-Oncology Centers Network Data Coordinating Center (http://opso.cancer.gov/data).

Cell Lines: MCF-10A and MDA-MB-231 cell lines used for the pilot study are available at the PS-OC Bioresource Core Facility (http://opso.cancer.gov/pbcf)

Competing financial interests: The authors declare no competing financial interests.

License: This work is licensed under a Creative Commons

Attribution-NonCommercial-NoDerivs 3.0 Unported License. To view a copy of this license, visit http://creativecommons.org/licenses/by-nc-nd/3.0/

How to cite this article: The Physical Sciences-Oncology Centers Network. A physical sciences network characterization of non-tumorigenic and metastatic cells. Sci. Rep. 3, 1449; DOI:10.1038/srep01449 (2013). 
David B. Agus', Jenolyn F. Alexander², Wadih Arap ${ }^{3}$, Shashanka Ashili ${ }^{4}$, Joseph E. Aslan ${ }^{5,6}$, Robert H. Austin 7 , Vadim Backman ${ }^{8}$, Kelly J. Bethel ${ }^{9}$, Richard Bonneau ${ }^{10}$, Wei-Chiang Chen ${ }^{11}$, Chira Chen-Tanyolac ${ }^{12}$, Nathan C. Choi ${ }^{1}$, Steven A. Curley ${ }^{13}$, Matthew Dallas $^{11}$, Dhwanil Damania ${ }^{8}$, Paul C.W. Davies ${ }^{14}$, Paolo Decuzzi ${ }^{2}$, Laura Dickinson ${ }^{11}$, Luis Estevez-Salmeron ${ }^{12}$, Veronica Estrella ${ }^{15}$, Mauro Ferrari ${ }^{2}$, Claudia Fischbach ${ }^{16}$, Jasmine Foo ${ }^{17}$, Stephanie I. Fraley ${ }^{11}$, Christian Frantz ${ }^{18}$, Alexander Fuhrmann ${ }^{19}$, Philippe Gascard $^{12}$, Robert A. Gatenby ${ }^{15}$, Yue Geng ${ }^{16}$, Sharon Gerecht ${ }^{11}$, Robert J. Gillies ${ }^{15}$, Biana Godin ${ }^{2}$, William M. Grady ${ }^{20,21}$, Alex Greenfield $^{10}{ }^{\text {, Courtney Hemphill }}{ }^{4}$, Barbara L. Hempstead ${ }^{22}$, Abigail Hielscher ${ }^{11}$, W. Daniel Hillis ${ }^{1,23}$, Eric C. Holland ${ }^{24}$, Arig lbrahimHashim $^{15}$, Tyler Jacks ${ }^{25,26}$, Roger H. Johnson ${ }^{4}$, Ahyoung Joo ${ }^{1}$, Jonathan E. Katz ${ }^{1}$, Laimonas Kelbauskas ${ }^{4}$, Carl Kesselman ${ }^{27}$, Michael R. King ${ }^{16}$, Konstantinos Konstantopoulos ${ }^{11}$, Casey M. Kraning-Rush ${ }^{16}$, Peter Kuhn ${ }^{28}$, Kevin Kung ${ }^{29}$, Brian Kwee ${ }^{16}$, Johnathon N. Lakins $^{18}$, Guillaume Lambert ${ }^{7}$, David Liao ${ }^{12}$, Jonathan D. Licht ${ }^{30}$, Jan T. Liphardt ${ }^{31,32}$, Liyu Liu ${ }^{7}$, Mark C. Lloyd ${ }^{15,33}$, Anna Lyubimova $^{29}$, Parag Mallick ${ }^{1,34}$, John Marko ${ }^{35}$, Owen J.T. McCarty ${ }^{5,6}$, Deirdre R. Meldrum ${ }^{4}$, Franziska Michor ${ }^{36}$, Shannon M. Mumenthaler ${ }^{1}$, Vivek Nandakumar ${ }^{4}$, Thomas V. O'Halloran ${ }^{37}$, Steve Oh ${ }^{12}$, Renata Pasqualini ${ }^{3}$, Matthew J. Paszek ${ }^{18}$, Kevin G. Philips ${ }^{5}$, Christopher S. Poultney ${ }^{10}$, Kuldeepsinh Rana ${ }^{16}$, Cynthia A. Reinhart-King ${ }^{16}$, Robert Ros ${ }^{19}$, Gregg L. Semenza ${ }^{38}$, Patti Senechal ${ }^{4}$, Michael L. Shuler $^{16,39}$, Srimeenakshi Srinivasan ${ }^{2}$, Jack R. Staunton ${ }^{19}$, Yolanda Stypula ${ }^{8}$, Hariharan Subramanian ${ }^{8}$, Thea D. Tlsty ${ }^{12}$, Garth W. Tormoen ${ }^{5}$, Yiider Tseng ${ }^{11}$, Alexander van Oudenaarden ${ }^{25,29}$, Scott S. Verbridge ${ }^{16 \dagger}$, Jenny C. Wan ${ }^{1}$, Valerie M. Weaver $^{18,40}$, Jonathan Widom ${ }^{35}$, Christine Will ${ }^{30}$, Denis Wirtz ${ }^{11}$, Jonathan Wojtkowiak ${ }^{15}$ \& Pei-Hsun Wu ${ }^{11}$

\footnotetext{
'Center for Applied Molecular Medicine, Keck School of Medicine, University of Southern California, Los Angeles, CA 90033, ${ }^{2}$ Department of Nanomedicine, The Methodist Hospital Research Institute, Houston, TX 77030, ${ }^{3}$ David H. Koch Center, The University of Texas M.D. Anderson Cancer Center, Houston, TX 77030, ${ }^{4}$ Center for Biosignatures Discovery Automation, Biodesign Institute, Arizona State University, Tempe, AZ 85287, ${ }^{5}$ Department of Biomedical Engineering, School of Medicine, Oregon Health \& Science University, Portland, OR 97239, ${ }^{6}$ Department of Cell and Developmental Biology, School of Medicine, Oregon Health \& Science University, Portland, OR 97239, ${ }^{7}$ Department of Physics, Princeton University, Princeton, NJ 08544, ${ }^{8}$ Biomedical Engineering Department, Northwestern University, Evanston, IL 60208, ${ }^{9}$ Department of Pathology, Scripps Clinic, La Jolla, CA 92037, ${ }^{10}$ Department of Biology, Department of Computer Science, New York University, New York, NY 10003, ${ }^{11}$ Department of Chemical and Biomolecular Engineering, The Johns Hopkins University, Baltimore, MD $21218,{ }^{12}$ Department of Pathology and UCSF Helen Diller Family Comprehensive Cancer Center, University of California at San Francisco, San Francisco, CA, 94143, ${ }^{13}$ Department of Surgical Oncology, The University of Texas M. D. Anderson Cancer Center, Houston, TX 77030, ${ }^{14}$ Beyond Center for Fundamental Concepts in Science, Arizona State University, Tempe, AZ 85287, ${ }^{15}$ Departments of Radiology and Integrated Mathematical Oncology, H. Lee Moffitt Cancer Center and Research Institute, 12902 Magnolia Drive, Tampa, FL 33612, ${ }^{16}$ Department of Biomedical Engineering, Cornell University, Ithaca, NY 14853, ${ }^{17}$ School of Mathematics, University of Minnesota Twin Cities, Minneapolis, MN 55455, ${ }^{18}$ Department of Surgery and Center for Bioengineering and Tissue Regeneration, University of California at San Francisco, San Francisco, CA 94143, ${ }^{19}$ Department of Physics, Arizona State University, Tempe, AZ 85287, ${ }^{20}$ Clinical Research Division, Fred Hutchinson Cancer Research Center, Seattle, WA 98109, ${ }^{21}$ Department of Medicine, University of Washington, Seattle, WA 98195, ${ }^{22}$ Department of Medicine, Weill Cornell Medical College, New York, NY 10065, ${ }^{23}$ Applied Minds, Inc. Glendale, CA 91201, ${ }^{24}$ Cancer Biology and Genetics Program, Department of Neurosurgery, Memorial Sloan-Kettering Cancer Center, New York, NY 10065, ${ }^{25}$ Department of Biology, Massachusetts Institute of Technology, Cambridge, MA $02139,{ }^{26}$ Koch Institute for Integrative Cancer Research and Howard Hughes Medical Institute, Massachusetts Institute of Technology, Cambridge, MA 02139, ${ }^{27}$ Information Sciences Institute, University of Southern California, Los Angeles, 90292, ${ }^{28}$ Department of Cell Biology, The Scripps Research Institute, La Jolla, CA 92037, ${ }^{29}$ Department of Physics, Massachusetts Institute of Technology, Cambridge, MA 02139, ${ }^{30}$ Division of Hematology/Oncology, Robert H. Lurie Comprehensive Cancer Center, Feinberg School of Medicine, Northwestern University, Chicago, IL 606 11, ${ }^{31}$ Department of Physics, Biophysics Graduate Group, University of California - Berkeley, Berkeley, CA 94720, ${ }^{32}$ Physical Biosciences Division, Lawrence Berkeley National Laboratory, Berkeley, CA 94720, ${ }^{33}$ Department of Analytic Microscopy, H. Lee Moffitt Cancer Center and Research Institute, 12902 Magnolia Drive, Tampa, FL 33612, ${ }^{34}$ Department of Radiology, School of Medicine, Stanford University, Stanford, CA 94305, ${ }^{35}$ Department of Biochemistry, Molecular Biology, and Cell Biology, Northwestern University, Evanston, IL 60208, ${ }^{36}$ Department of Biostatistics and Computational Biology, Dana-Farber Cancer Institute, and Department of Biostatistics, Harvard School of Public Health, Boston, MA 021 15, ${ }^{37}$ Department of Chemistry, Chemistry of Life Processes Institute, Northwestern University, Evanston, IL 60208, ${ }^{38}$ Vascular Program, Institute of Cell Engineering and McKusickNathans Institute of Genetic Medicine and the Departments of Pediatrics, Medicine, Oncology, Radiation Oncology, and Biological Chemistry, The Johns Hopkins University School of Medicine, Baltimore, MD 21205, ${ }^{39}$ School of Chemical and Biomolecular Engineering, Cornell University, lthaca, NY $14853,{ }^{40}$ Department of Anatomy, Department of Bioengineering and Therapeutic Sciences, Eli and Edythe Broad Center of Regeneration Medicine and Stem Cell Research at UCSF, and Helen Diller Comprehensive Cancer Center, University of California at San Francisco, San Francisco, CA 94143.
}

${ }^{\dagger}$ Current address: School of Biomedical Engineering and Sciences, Virginia Tech-Wake Forest University, Blacksburg, VA, 24061.

:Current address: Department of Chemical Engineering, University of Florida, Gainesville, FL, 32611. 